\title{
ORIGINAL ARTICLE Inbreeding depression across a nutritional stress continuum
}

\begin{abstract}
MF Schou ${ }^{1}$, V Loeschcke ${ }^{1}$ and TN Kristensen ${ }^{2}$
Many natural populations experience inbreeding and genetic drift as a consequence of nonrandom mating or low population size. Furthermore, they face environmental challenges that may interact synergistically with deleterious consequences of increased homozygosity and further decrease fitness. Most studies on inbreeding-environment (I-E) interactions use one or two stress levels, whereby the resolution of the possible stress and inbreeding depression interaction is low. Here we produced Drosophila melanogaster replicate populations, maintained at three different population sizes (10, 50 and a control size of 500$)$ for 25 generations. A nutritional stress gradient was imposed on the replicate populations by exposing them to 11 different concentrations of yeast in the developmental medium. We assessed the consequences of nutritional stress by scoring egg-toadult viability and body mass of emerged flies. We found: (1) unequivocal evidence for I-E interactions in egg-to-adult viability and to a lesser extent in dry body mass, with inbreeding depression being more severe under higher levels of nutritional stress; (2) a steeper increase in inbreeding depression for replicate populations of size 10 with increasing nutritional stress than for replicate populations of size 50; (3) a nonlinear norm of reaction between inbreeding depression and nutritional stress; and (4) a faster increase in number of lethal equivalents in replicate populations of size 10 compared with replicate populations of size 50 with increasing nutritional stress levels. Our data provide novel and strong evidence that deleterious fitness consequences of I-E interactions are more pronounced at higher nutritional stress and at higher inbreeding levels.
\end{abstract}

Heredity (2015) 115, 56-62; doi:10.1038/hdy.2015.16; published online 18 March 2015

\section{INTRODUCTION}

Natural populations are exposed to variable and often stressful environments (Hoffmann and Parsons, 1991; Bijlsma and Loeschcke, 2005) in which stressors such as extreme temperatures, low water availability and parasites strongly influence their distribution and fitness (Andrewartha and Birch, 1954). Furthermore, many populations in nature become inbred and lose genetic variation as they experience reductions in population size (Crnokrak and Roff, 1999). In consequence, they likely experience a decreased fitness (that is, inbreeding depression) and reduced evolutionary potential compared with outbred or large populations (Hoffmann and Parsons, 1991; Falconer and Mackay, 1996; Hoffmann and Willi, 2008; Reed et al., 2012; Kristensen et al., 2015). Thus, an understanding of the effects of environmental stress, inbreeding and genetic drift and potential interactions between them on fitness are of foremost importance if we are to apprehend the ecological and evolutionary challenges faced by natural populations (Crnokrak and Roff, 1999; Cheptou and Donohue, 2011; Bijlsma and Loeschcke, 2011; Reed et al., 2012). Inbreeding effects have been investigated not only at benign but also at stressful environmental conditions in a number of studies and in meta-analyses and reviews (Armbruster and Reed, 2005; Fox and Reed, 2011). A majority of these studies show that inbreeding depression is more pronounced in stressful compared with benign environments, whereby the effects of inbreeding and environmental stress are synergistic (Armbruster and Reed, 2005; Fox and Reed, 2011).

There are however conflicting results and opinions as to the generality and causation of inbreeding-environment (I-E) interactions
(Reed et al., 2012). I-E interactions have traditionally been viewed as a special case of genotype-environment interactions, for example, observed as a distinct change in gene expression across varying environmental conditions in inbred and outbred populations (Kristensen et al., 2006, 2010). The changed gene expression profile can lead to an increased inbreeding depression in stressful environments by three nonexclusive mechanisms: (1) changed expression of deleterious alleles as a consequence of environmental stress (that is, conditional deleterious alleles); (2) reduced stress resistance (increased environmental sensitivity) as a consequence of expression of deleterious alleles; and (3) increased phenotypic variance $\left(\mathrm{CV}^{2}\right)$ that increases the opportunity for overall fitness to decrease (Waller et al., 2008; Bijlsma and Loeschcke, 2011; Reed et al., 2012).

The effect of inbreeding on fitness has often been found to be dependent on the rate of inbreeding, as a slower rate may effectuate the removal of recessive deleterious alleles through natural selection (that is, purging) (Hedrick, 1994; Wang et al., 1999). Purging in a given stressful environment may render a population less susceptible to that specific stress (Bijlsma et al., 1999). Although this assumes environment-specific purging, purging of deleterious alleles that have deleterious effects across different environmental conditions could decrease the effects of I-E interactions in novel environments. This makes the rate of inbreeding important to consider in studies on I-E interactions.

Studies on fitness effects of I-E interactions typically investigate one or two levels of environmental stress, often simply contrasting a benign environment to a stressful environment (see references in Armbruster and Reed, 2005; Fox and Reed, 2011; Supplementary 
Table S1). The low resolution of the stress dimension raises a number of issues as the stress level and stress type vary between studies. For example, the relationship between stress and inbreeding depression may be a stressor and trait-specific phenomenon. Thus, a lack of strong effects of I-E interactions on fitness traits might stem from low environmental stress levels and nonlinear norms of reaction (Fox and Reed, 2011). To approach these issues it is necessary to assess the effects of inbreeding across a continuous range of stress levels. This would enable determination of the stress level at which I-E interactions appear and the shape of the change in inbreeding depression with increasing stress.

In this study we aimed to estimate the norm of reaction of I-E interactions across a continuous range of stress levels and two inbreeding levels $(\mathrm{F}=0.31$ and 0.84$)$ obtained at different rates. We maintained replicate populations of Drosophila melanogaster at three different population sizes (10,50 and 500), reflective of census or effective population sizes of many domestic and natural populations (Palstra and Ruzzante, 2008; Leroy et al., 2013). We measured egg-toadult viability and dry body mass of flies that have developed at 11 different nutritional levels (ranging from benign to highly stressful), for replicate populations of the three different population sizes. Availability and quality of food for natural populations varies with season, altitude, latitude and habitat type (Suzuki, 1998; Johnson and Sherry, 2001; Cotton, 2006), whereby nutritional stress represents a natural continuous stressor in Drosophila (Markow et al., 1999), and is an ecologically relevant framework for investigating I-E interactions.

\section{MATERIALS AND METHODS}

\section{Founding population}

A D. melanogaster population was established in 2010 from the virgin offspring of 589 females caught at the Danish peninsula of Jutland (for further details see Schou et al., 2014). Each of the females contributed to the mass bred population with five male and five female offspring. The mass bred population was maintained with the approximate population size of 6000 at $25^{\circ} \mathrm{C}$ in a 12:12 light/dark photoperiod for two generations before the establishment of replicate populations. Unless otherwise indicated, the medium used for maintenance of flies is a standard oatmeal-sugar-yeast-agar Drosophila medium (Table 1: control medium).

\section{Inbreeding procedure}

A total of 10, 6 and 3 replicate populations of population size 10 (N10), 50 (N50) and 500 (N500), respectively, were established using virgin flies from the mass bred population. These replicate populations were maintained at the specified population sizes and with equal sex ratios for 25 generations.

Table 1 The composition of the 11 different developmental media

\begin{tabular}{lccc}
\hline Medium & Yeast $\left(g l^{-1}\right)$ & Oatmeal $\left(g l^{-1}\right)$ & Sugar $\left(g l^{-1}\right)$ \\
\hline Control & 60 & 30 & 40 \\
Medium 1 & 10.6 & 0 & 0 \\
Medium 2 & 9.4 & 0 & 0 \\
Medium 3 & 8.3 & 0 & 0 \\
Medium 4 & 7.1 & 0 & 0 \\
Medium 5 & 6.0 & 0 & 0 \\
Medium 6 & 4.9 & 0 & 0 \\
Medium 7 & 3.7 & 0 & 0 \\
Medium 8 & 2.6 & 0 & 0 \\
Medium 9 & 1.4 & 0 & 0 \\
Medium 10 & 0.3 & 0 & 0
\end{tabular}

All media contained the same amounts of agar $\left(16 \mathrm{gl}^{-1}\right)$, nipagin $\left(12 \mathrm{ml}^{-1}\right)$ and acetic acid $\left(1.2 \mathrm{mll}^{-1}\right)$. The control medium is used in Drosophila experiments as an ad libitum food source, and therefore contains much more nutrition than needed for successful development.
We controlled rearing density during development and maintained 1:1 sex ratio. A succeeding generation was produced by $5-8$-day-old flies that laid eggs for a $12 \mathrm{~h}$ period followed by a $24 \mathrm{~h}$ period of egg laying in another set of bottles. Low-density bottles were used to establish the next generation. Each replicate $\mathrm{N} 10$ population was maintained in a $27 \mathrm{ml}$ vial with $7 \mathrm{ml}$ medium, each replicate N50 population was maintained in a $100 \mathrm{ml}$ glass bottle with $35 \mathrm{ml}$ medium and each replicate N500 population was maintained in ten $100 \mathrm{ml}$ glass bottles, each with 50 flies and $35 \mathrm{ml}$ medium. To approximately maintain the level of inbreeding reached at generation 25, replicate N10 and N50 populations were kept in a $100 \mathrm{ml}$ glass bottle with $35 \mathrm{ml}$ food, and flushed to a population size of 250 , whereas replicate N500 populations were distributed in three $100 \mathrm{ml}$ glass bottles and flushed to a population size of 750 . We expect only minor increases in the level of inbreeding as a consequence of the increased population size $\left(\Delta F_{\mathrm{N} 10}=0.006\right.$ and $\left.\Delta F_{\mathrm{N} 50}=0.027\right)$, and have disregarded this in the estimation of the inbreeding level. The replicate populations were kept in a climate chamber with a daily change in temperature following a Gaussian function (a bell-shaped curve; for details see the control regime in Schou et al., 2014). The night time temperature was $23.5^{\circ} \mathrm{C}$, from which the temperature increased and reached the daily temperature peak of $27.5^{\circ} \mathrm{C}$. The average temperature was $24.8^{\circ} \mathrm{C}$. The light rhythm followed the same daily pattern as the temperature, with a midday peak and gradual decrease during night.

\section{Experimental design}

To measure fitness components at different nutritional stress levels we assessed egg-to-adult viability of the 19 replicate populations in the control medium at which they were originally reared (containing oatmeal, sugar and yeast) and in 10 media only containing yeast, but differing in yeast concentrations (Table 1). The progressive dilution of the yeast concentration decreases the available protein per volume medium, limiting the amount of protein readily obtained by the larvae, and thereby imposes a stress on nutritional requirements for the development (Economos and Lints, 1985). The yeast concentrations used were based on results from pilot experiments. The criterion was that nutritional stress, observed as a decrease in egg-to-adult viability, should be imposed on replicate N10 populations at the least stressful medium compared with the control medium. The flies did not experience nutritional stress during the inbreeding procedure as they were reared at controlled densities with ad libitum food (control medium), and thus the imposed nutritional stress is considered to be a novel stress. All media were produced with an automatized cooking program in a mediaclave (Integra Biosciences, Zizers, Switzerland), ensuring homogeneity in the production pipeline and avoiding any yeast growth in the media (for details see Supplementary Text S1).

The parental flies used for egg production developed at a density of 40 eggs (in a $27 \mathrm{ml}$ vial containing $7 \mathrm{ml}$ medium) to avoid density-dependent maternal effects. To ensure random sampling of eggs from the parental flies, and to avoid any media or microorganisms being present on the eggs when transferred to the developmental medium, we used an egg collection method with a $2.5 \%$ agar medium as described in Schou (2013) (see below for a brief description). The parental flies (between 3 and 6 days old) of each replicate population produced eggs from 1800 to $0800 \mathrm{~h}$ in two $300 \mathrm{ml}$ plastic bottles each containing $50 \mathrm{ml}$ agar medium and a pile of yeast-paste. The following day, flies were maintained on standard Drosophila medium, whereas eggs from the preceding bottles of each replicate population were washed in a sucrose solution, randomized and transferred to a filter paper. Eggs were then transferred from the filter paper to vials containing the different media, such that each vial contained exactly 20 eggs and such that 10 replicate vials were collected for each medium for each replicate population. A total of 41800 eggs were distributed to 2090 vials. Eggs were picked 4 days in a row, such that for the 11 media, approximately the same number of replicates was completed for a given replicate population at a given day. The egg transferring was done simultaneously for all replicate populations. During development, the vials with eggs were kept at $23.5 \pm 1{ }^{\circ} \mathrm{C}$, and their positions were randomized on a daily basis. Every second day, the emerged flies of a vial were transferred to an empty vial, sexed, counted and frozen for the assessment of dry body mass. The control medium (also containing sugar and oatmeal) was omitted from all analyses along with 
medium 10 in which no replicate population produced more than two survivors.

Dry body mass of $15.5 \pm 1.7$ males per replicate population per treatment (for exact numbers see Supplementary Table S2) was measured with a Sartorius Laboratory Balance (type MC5, Göttingen, Germany). The flies were dried at $60^{\circ} \mathrm{C}$ for $24 \mathrm{~h}$. To avoid large amounts of water being absorbed by the dry flies in the process of weighing them, we transferred one sample at a time to a low humidity box containing silica gel, from which the flies were transferred individually to the balance. Pooling of all data showed a minor but significant positive correlation (Pearson's $r=0.05 ; t(2823)=2.47, P<0.01$ ) between dry body mass and the order in which the flies were obtained and weighed from the low humidity container. Subsequently, the dry body mass was corrected, by estimating the effect of water absorbance with a linear model and adjusting the dry body mass accordingly.

\section{Estimating stress levels}

We transformed the nutritional stress level (yeast concentration) into a standardized measure of stress, to enable comparison of the phenotypic response in this study with results from studies investigating other stressors. Tradeoffs between body size and developmental time (Nunney, 1996; Sørensen and Loeschcke, 2004) suggest that dry body mass is a more indirect measure of fitness compared with egg-to-adult viability, especially in this study where flies are submitted to nutritional stress that affects both body size and developmental time. For this reason, and as survival has been used as a measure of stress in comparable studies (Armbruster and Reed, 2005; Fox and Reed, 2011), we chose to use fitted egg-to-adult viability of replicate N500 populations as a measure of stress level of each yeast concentration. The stress level of a given medium $i$ was estimated as the proportional decrease in survival compared with medium one, stress level $_{i}=1-\left(\right.$ fitted survival $_{\text {N500 (medium } i)} /$ fitted survival $_{\mathrm{N} 500 \text { (medium 1) }}$ ).

The fitted values of egg-to-adult viability for the replicate N500 populations were obtained with a generalized linear mixed model with a logistic link using the R package lme4 (Bates et al., 2014; R Core Team, 2015). Yeast concentration was $\log$ transformed to provide a better fit of the model.

\section{Inbreeding depression for egg-to-adult viability and dry body mass} For each replicate inbred population we estimated inbreeding depression $(\delta)$ in egg-to-adult viability and dry body mass, as the proportional reduction in survival or dry body mass compared with the mean of the replicate control populations (Fox and Reed, 2011), $\delta=\left(\right.$ mean $_{\mathrm{N} 500}-$ mean $\left._{\text {inbred }}\right) /$ mean $_{\mathrm{N} 500}$.

We assessed whether inbreeding depression of egg-to-adult viability and dry body mass increased with stress and whether this increase was dependent on breeding regime using generalized linear mixed models in the $\mathrm{R}$ package lme4 (Bates et al., 2014). The full models contained the fixed effects stress, estimated from egg-to-adult viability (see above), and breeding regime (N10 and N50) as well as their interaction. In the full model stress was included as a quadratic effect to test whether this would improve the fit. To evaluate which random effects to incorporate, we included all fixed effects in the full model, and assessed the validity of the different random effects using Akaike information criterion (Zuur et al., 2009). The random effects evaluated were (1) random intercept of replicate populations, (2) random intercept and slope of replicate populations and (3) random intercept and quadratic slopes of replicate populations (Schielzeth and Forstmeier, 2009). We assessed the fixed effects by using a likelihood ratio test to compare the full model with a reduced model and thus obtaining the observed $\log$-likelihood ratio statistic $\Lambda$ for a given fixed effect. We used parametric bootstrapping $(n=10000)$ to obtain the null distribution of $\Lambda$ under the reduced model. $P$-values were calculated as the proportion of empirical $\Lambda$ in the null distribution that were greater than the observed $\Lambda$. This approach was necessary as random effects can distort the test statistic distribution $(\Lambda)$ from the typically assumed $\chi^{2}$ distribution. The assumptions of a normal distribution of the residuals and homogeneity of variances were fulfilled.

As the interaction between breeding regime and stress was significant in the analysis of inbreeding depression of egg-to-adult viability, we split the data set and investigated the effect of stress in each breeding regime by performing model comparisons as above. We found no significant interaction between breeding regime and stress in the analysis of inbreeding depression of dry body mass and therefore continued with sequential model reduction and model comparison as above.

\section{Lethal equivalents}

As a prerequisite for calculating the number of haploid lethal equivalents, we estimated the expected inbreeding level (Crow and Kimura, 1970) after $t$ generations $\left(f_{t}\right)$ by assuming $f_{0}=0$ and the ratio between effective population size $(\mathrm{Ne})$ and the census size $(N)$ being $N e=2 / 3 N$ (Buri 1956; Nunney 1993 (lottery polygyny and Poison distributed offspring per mating)) using:

$$
f_{t}=f_{t-1}+\frac{\left(1-2 f_{t-1}+f_{t-2}\right)}{2 N_{e}}
$$

The estimated inbreeding levels were $F_{\mathrm{N} 10}=0.84$ and $F_{\mathrm{N} 50}=0.31$. The number of haploid lethal equivalents, $B$, is a measure of the rate by which fitness changes with increasing inbreeding level (Morton et al., 1956). We calculated $B$ for replicate inbred populations by the formula:

$$
B=-\frac{1}{f_{t}} \ln \left(\frac{w_{f}}{w_{o}}\right)
$$

Here, $w_{\mathrm{f}}$ is the average fitness of the replicate inbred population and $w_{\mathrm{o}}$ is the average fitness of replicate outbred populations, for the given medium. A fitness estimate of zero resulted in negative infinite estimates of $B$. We considered two approaches to solve this problem: (1) removal of estimates of zero and (2) setting estimates of zero equal to 0.005 . We observed no difference in the conclusions based on these two methods (data not shown) and proceeded with approach 2. The data on lethal equivalents across stress levels did not fulfil the assumption of normality of residuals for standard parametric analysis. Therefore, we calculated a linear regression coefficient for each replicate population. Assuming no relationship between stress and lethal equivalents, the probability of obtaining a positive slope is as likely as obtaining a negative slope. We therefore evaluated the chance of obtaining the given proportion of positive slopes within breeding regime using a Bernoulli trial (or binomial trial). To investigate whether the slopes of N10 and N50 differed we performed a randomization test in which we randomized replicate populations across population sizes $(n=10000)$. The difference between the regression coefficients of the two groups was chosen as test statistic. As data were not normally distributed and to put emphasis on general trends, we estimated the regression coefficients with a two-quantile (median) regression (Koenker and D'Orey, 1987) implemented in the $\mathrm{R}$ package 'quantreg' (Koenker, 2013). After establishing the null distribution of the difference in regression coefficients between the two groups, we performed a two-tailed test to assess the $P$-value for the observed difference in regression coefficients. The results from the analysis of lethal equivalents led to the same conclusions when using $\mathrm{Ne} / \mathrm{N}$ ratios from $\sim$ zero to one (data not shown).

\section{RESULTS}

The change in egg-to-adult viability with decreasing yeast concentration across breeding regimes is presented in Figure 1. In the analysis of inbreeding depression of egg-to-adult viability, the most appropriate random components were random intercept and quadratic slopes of replicate populations, illustrating the large variation in the curvature between replicate populations (Figure 2). Including stress as a quadratic component significantly improved the fit $(\Lambda=6.62$, $P=0.048)$ of the model. We found a significant interaction between breeding regime and stress on inbreeding depression $(\Lambda=8.63$, $P=0.027$; Figure 2), demonstrating that replicate N10 populations had a higher rate of increase in inbreeding depression with increasing stress compared with replicate N50 populations. When outbred populations experienced a decrease in fitness of $30 \%$ (stress $=0.3$ ), N10 populations had a fitness of $26 \%$ of the outbred populations and N50 populations had a fitness of $69 \%$ of the outbred populations. The observed curvature was very different between breeding regimes (Figure 2), and therefore we split the data set according to breeding regime and reassessed whether the use of stress as a quadratic term 
improved the fit. The quadratic term improved the fit for the replicate N10 populations $(\Lambda=5.42, P=0.044)$ but not for the replicate N50 populations $(\Lambda=0.18, P=0.677)$. The increase in inbreeding depression with increasing stress (I-E interaction) was significant for replicate N10 populations $(\Lambda=32.58, \quad P<0.001)$ and in replicate N50

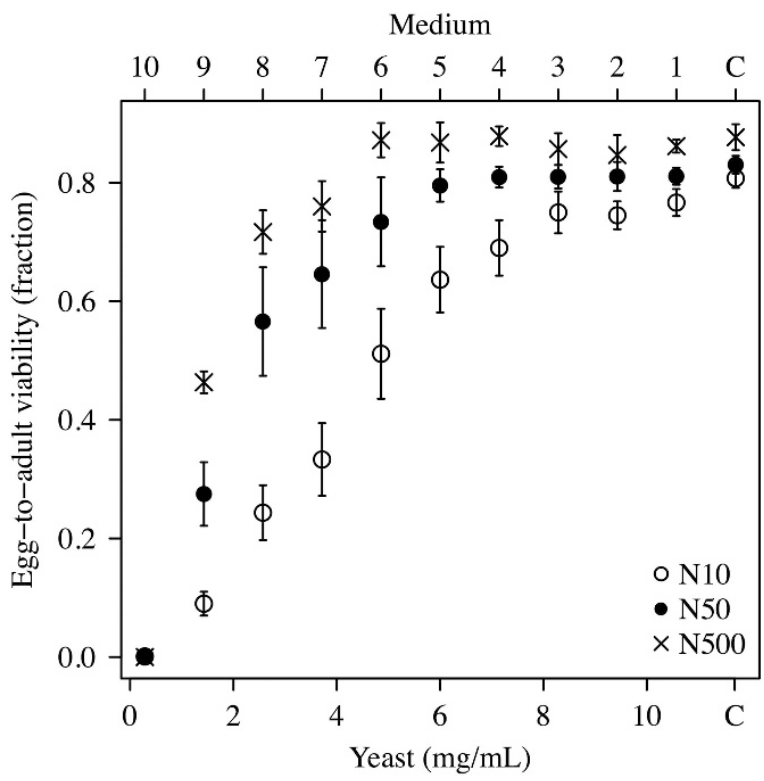

Figure 1 Average egg-to-adult viability and standard errors of replicate N10 $(n=10), \mathrm{N} 50(n=6)$ and $\mathrm{N} 500(n=3)$ populations in the control medium (C) and in media with different yeast concentrations. Medium numbers are presented on the upper axis.

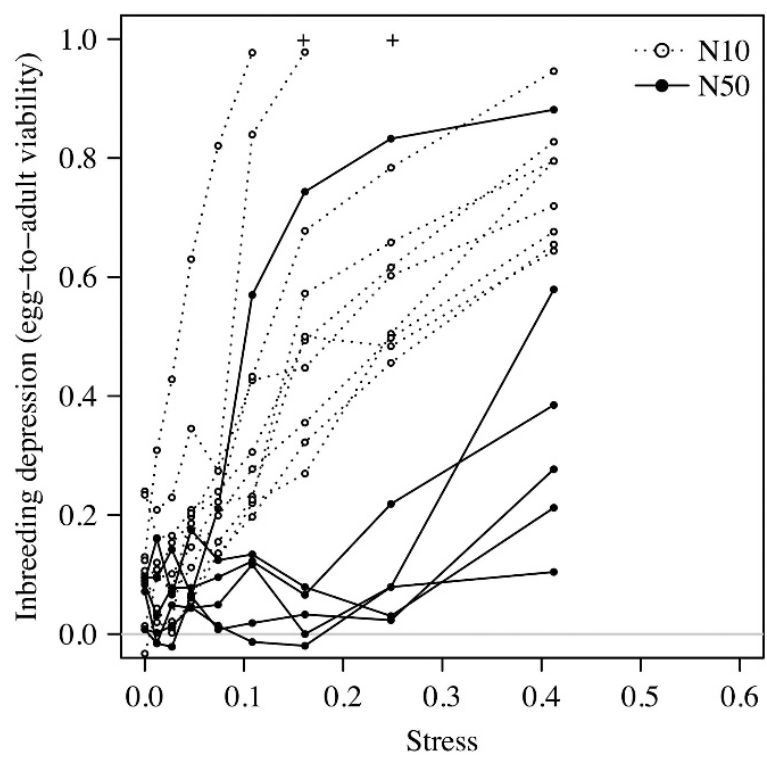

Figure 2 Inbreeding depression of egg-to-adult viability in replicate N10 $(n=10)$ and $\mathrm{N} 50(n=6)$ populations at a range of stress levels. Stress level for medium $i$ was calculated as 1 - (fitted survival $\left.\right|_{N 500}$ (medium in) $/$ itted survival $_{N 500}$ (medium 1)). Replicate N10 populations display a steep initial increase in inbreeding depression; however, at high stress levels the rate of increase decreases. Replicate N50 populations show a different norm of reaction, but with large differences between replicate populations. Two replicate N10 populations reached a survival of zero, as indicated with ' + ' for each replicate population. populations $(\Lambda=8.60, P=0.024)$. The increase in inbreeding depression of two replicate N10 populations reached an inbreeding depression of one (corresponding to a survival of zero, Figure 2) that may result in a curvature of the average increase in inbreeding depression with increasing stress. For this reason we excluded estimates of inbreeding depression of one and repeated the analysis presented above, and this led to the same conclusions.

The large range in availability of developmental nutrition resulted in flies ranging in dry body mass from 0.03 to $0.31 \mathrm{mg}$ (Figure 3 ). In the analysis of the effect of stress on inbreeding depression in dry body mass, we included a random intercept of replicate populations. A quadratic relationship between stress and inbreeding depression did not improve the fit $(\Lambda=0.54, P=0.785)$. There was no significant interaction between breeding regime and stress $(\Lambda=1.16, P=0.293$; Figure 4) and no significant difference between breeding regimes in dry body mass $(\Lambda=1.92, P=0.191)$. However, across all replicate inbred populations we found a significant increase in inbreeding depression at higher stress levels $(\Lambda=14.91, P<0.001$; Figure 4$)$.

All replicate populations had a positive regression coefficient of lethal equivalents across increasing stress levels. This resulted in an overall significant increase in lethal equivalents when increasing stress for N50 $(P(x=6)=0.016)$ and N10 $(P(x=10)<0.001)$ (Figure 5). Replicate N10 populations had a significantly larger increase in lethal equivalents with increasing stress than the replicate N50 populations $\left(\right.$ slope $_{\mathrm{N} 10}=4.38 \pm 0.63 ;$ slope $_{\mathrm{N} 50}=1.52 \pm 1.12 ; P=0.045 ;$ Figure 5$)$.

\section{DISCUSSION}

The primary aim of this study was to investigate I-E interactions across a range of stress levels and to test how I-E interactions are affected by the population size at which replicate populations were reared. We found clearcut evidence that nutritional stress increases inbreeding depression for egg-to-adult viability and that the rate of this increase is dependent on the breeding regime. The rate of increase in inbreeding depression decreased with increasing stress for replicate N10 populations, whereby the norm of reaction was nonlinear. Higher stress levels did not increase inbreeding depression in dry body mass to the same

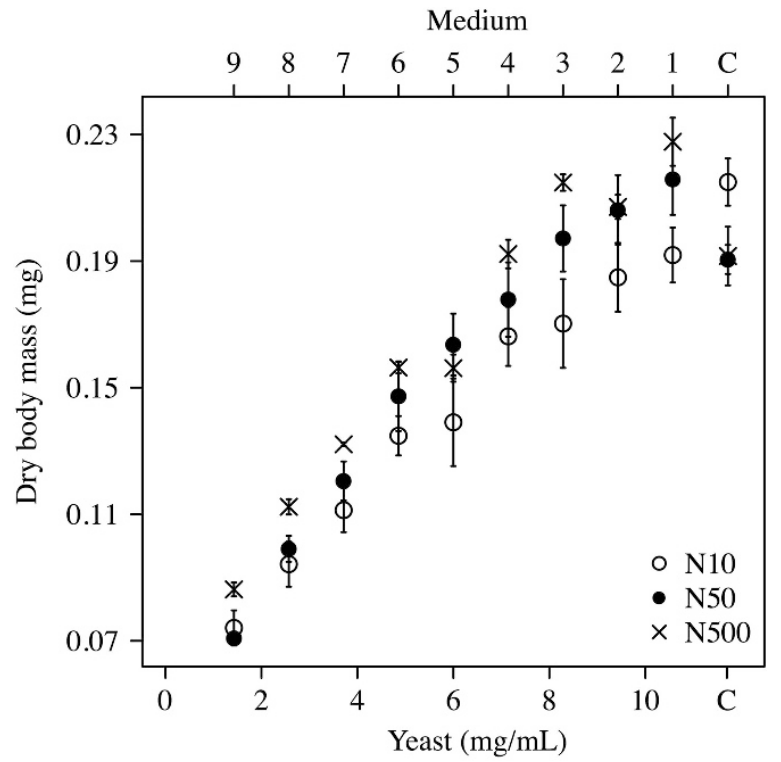

Figure 3 Average dry body mass and standard errors of replicate N10 $(n=10), \mathrm{N} 50(n=6)$ and $\mathrm{N} 500(n=3)$ populations developed in the control medium (C) and in different yeast concentrations. 
degree as for egg-to-adult viability. We also estimated the number of lethal equivalents, a standardized measure of inbreeding depression. When the stress increased, replicate N10 populations experienced a steeper increase in lethal equivalents than N50 populations.

Increasing stress resulted in large increases in inbreeding depression for egg-to-adult viability in replicate $\mathrm{N} 10$ and N50 populations. A quadratic relationship between stress level and inbreeding depression improved the fit for replicate N10 populations, whereas a linear relationship gave the best fit for the replicate N50 populations. Thus, we found not only unequivocal evidence for an I-E interaction but we also show large effects of demographic history on the norm of reaction. The high number of stress levels used in the study was crucial for enabling a detection of the nonlinear trend and the finding may have large implications for our understanding of inbreeding

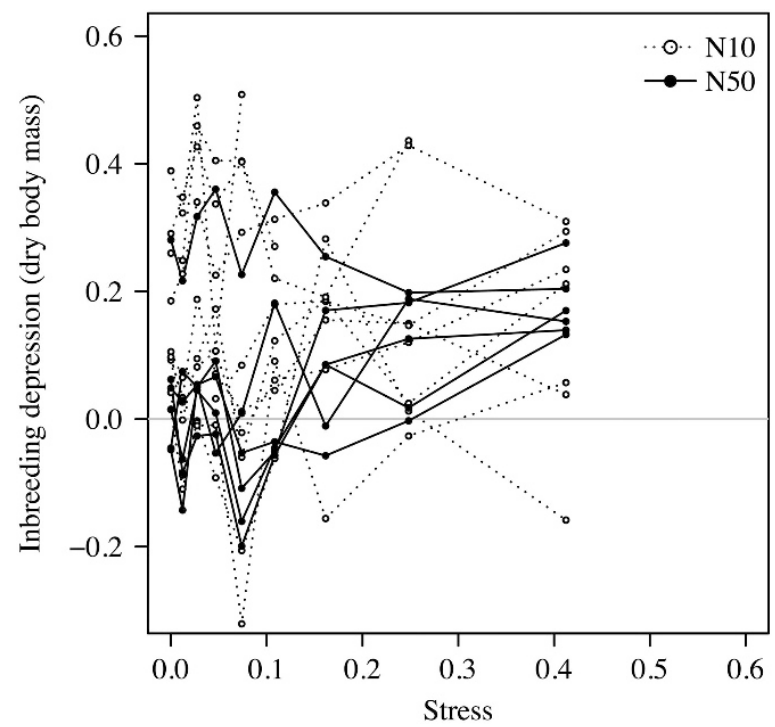

Figure 4 Inbreeding depression of dry body mass in replicate N10 $(n=10)$ and N50 $(n=6)$ populations at a range of stress levels. Stress level for medium $i$ was calculated as 1 - (fitted survival $\left.\right|_{N 500}$ (medium i)/fitted survival $_{\text {N500 (medium 1)). }}$ depression in natural populations. The stress levels investigated in this study provide a clearcut impression of the change in inbreeding depression with increasing stress for the highly inbred N10 populations. Conversely, most of the replicate N50 populations did not reach the same high levels of inbreeding depression, and we can only speculate that replicate N50 populations would show a similar curvature as N10 populations at higher stress levels. This expectation is supported by one highly stressed replicate N50 population that displays the same curvature as replicate N10 populations at high stress levels (Figure 2). An increased number of deleterious alleles expressed under stress may explain I-E interactions (see Reed et al., 2012 for a review of hypotheses). The nonlinear norm of reaction found in this study for replicate N10 populations may be the result of an upper boundary being reached in the number of deleterious alleles becoming expressed with increasing stress levels.

Although the effects of I-E interactions on morphological traits have been investigated in several studies, the morphological traits studied previously tend to be closely associated with fitness (for example, seed mass or number of flowers) (Norman et al., 1995; Cheptou et al., 2000). Dry body mass is only indirectly associated with fitness through effects on, for example, reproductive output, developmental time and time to sexual maturity and may have an intermediate optimum. Based on the expectation that nutritional stress would decrease dry body mass (Wang and Clark, 1995), and have detrimental effects on the reproductive output of females, we chose to treat increased dry body mass as adaptive. Inbreeding depression was lower for dry body mass and the I-E interaction was less pronounced compared with eggto-adult viability. A lower inbreeding depression for a morphological trait not closely associated to fitness is expected as it is likely that the trait does not show the same directional dominance as life history traits (Falconer and Mackay, 1996).

In this study we used different ecologically relevant population sizes as a mechanism to manipulate the amount of random genetic drift and thereby the expected inbreeding level in our replicate populations. This approach mimics the process of inbreeding in many natural populations. However, this approach also results in two parameters, namely inbreeding rate and inbreeding level, being confounded. These parameters generally differ between studies on inbreeding and I-E

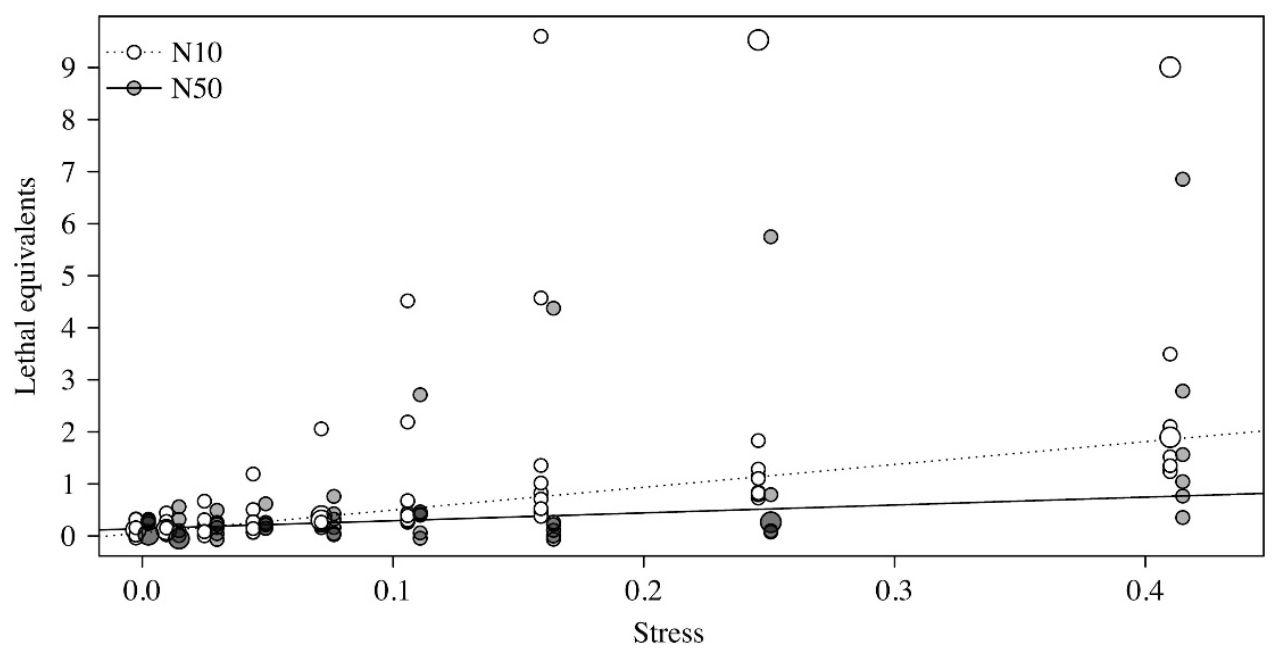

Figure 5 Number of lethal equivalents (calculated from egg-to-adult viability) of the replicate inbred populations across stress levels. Larger points indicate two replicate populations with the same number of lethal equivalents. The fitted lines were estimated with a two-quantile (median) regression to emphasize the general trends, and thus avoiding large influence of the extreme observations. 
interactions. For this reason, Armbruster and Reed (2005) and Fox and Reed (2011) used lethal equivalents (LE), a standardized measure of inbreeding depression, to standardize differences in inbreeding levels between studies. This enabled testing whether inbreeding depression increases with stress. We calculated LE and found a significant lower increase in LE with increasing stress of replicate N50 populations compared with N10 populations that, apart from their difference in inbreeding level, also differed in the rate of inbreeding. Thus, the rate of inbreeding cannot be neglected when standardizing the inbreeding level. We identified two nonexclusive explanations for the observed lower increase in LE with increasing stress of N50 compared with N10 populations. One possibility is a violation of the assumptions behind the standardization of inbreeding levels. The standardization assumes that independent deleterious loci have independent effects on fitness, whereby the logarithm of fitness will decrease linearly with increased inbreeding. There are obvious reasons why this assumption may be violated, for example, epistasis between deleterious loci that would deteriorate the fitness consequences of increased inbreeding. The other possibility is a more effective purging or balancing selection during the inbreeding of replicate N50 populations. The replicate inbred populations used in this experiment were inbred in an environment with ample nutritional resources, whereby purging of alleles that are lethal or mildly deleterious specifically in poor nutritional environments is unlikely. This suggests that a decreased rate of inbreeding, even under benign conditions, will result in a more effective removal of recessive mildly deleterious alleles that can become highly deleterious under stressful conditions. This possibility is to some extent in contrast to studies suggesting that purging is an environment-specific process. Comparing the rate of change in lethal equivalents with increasing stress in this study $\left(\right.$ slope $_{\mathrm{N} 10}=4.38 \pm 0.63$; slope $\left.e_{\mathrm{N} 50}=1.52 \pm 1.12\right)$ with that of the meta-analysis by Reed et al. (2012) (slope $=3.35 \pm 0.53)$ and with Enders and Nunney (2012) (slope $=1.56 \pm 0.39)$ reveals large discrepancies in the slope estimates. A part of the discrepancy between studies may stem from the use of replicate inbred populations with different demographic histories, such as different rates of inbreeding or violations of the assumptions behind LE.

We found indisputable evidence for I-E interactions that had drastic consequences for fitness components of replicate inbred populations. Quantity and quality of food is crucial for survival in nature and food resources are often limited (White, 1993). Our results suggest that fitness of inbred populations exposed to nutritional stress is more severely affected than that of less inbred populations. Nutritional stress and inbreeding interact synergistically and this interaction is likely to increase the extinction risk of small and endangered populations facing periods with food shortage. The difference in the relationship between stress level and expression of inbreeding depression between replicate N10 and N50 populations has implications for evolutionary biology and for the management strategies of threatened wild and domestic species. Minor stress levels deteriorated fitness of populations experiencing very small populations sizes $(N=10)$. Conversely, population sizes at an intermediate level $(N=50)$ may avoid I-E interactions up until stress levels of 0.25 (Figure 2). Stress levels in nature may in general be lower, but seasonal events such as cold winter, summer heat waves or food shortage can increase stress levels beyond 0.25 (see, for example, Enders and Nunney, 2012). The evolutionary dynamics and extinction risks of small populations will thus be highly dependent on the specific demographic history and the frequency of different stress levels in their environment.

\section{DATA ARCHIVING}

Data available from the Dryad Digital Repository (http://doi.org/ 10.5061/dryad.rf7s0).

\section{CONFLICT OF INTEREST}

The authors declare no conflict of interest.

\section{ACKNOWLEDGEMENTS}

We are grateful for the technical assistance offered by Doth Andersen, Søren Toft and Annemarie Højmark. This research was funded by the Graduate School of Science and Technology at Aarhus University to MFS, and by the Danish Natural Science Research Council with a frame grant to VL and a Sapere aude stipend to TNK (DFF-4002-00036).

Andrewartha HG, Birch LC (1954). The Distribution and Abundance of Animals. University of Chicago Press: Chicago.

Armbruster P, Reed DH (2005). Inbreeding depression in benign and stressful environments. Heredity 95: 235-242.

Bates D, Maechler M, Bolker B, Walker S (2014). Ime4: linear mixed-effects models using Eigen and S4. $R$ package version 1.1-5. http://cran.r-project.org/package=Ime4.

Bijlsma R, Bundgaard J, Van Putten WF (1999). Environmental dependence of inbreeding depression and purging in Drosophila melanogaster. J Evol Biol 12 $1125-1137$

Bijlsma R, Loeschcke V (2005). Environmental stress, adaptation and evolution: an overview. J Evol Biol 18: 744-749.

Bijlsma R, Loeschcke V (2011). Genetic erosion impedes adaptive responses to stressful environments. Evol App/ 5: 117-129.

Buri P (1956). Gene frequency in small populations of mutant Drosophila. Evolution 10: 367-402.

Cheptou P-O, Berger A, Blanchard A, Collin C, Escarre J (2000). The effect of drought stress on inbreeding depression in four populations of the Mediterranean outcrossing plant Crepis sancta (Asteraceae). Heredity 85: 294-302.

Cheptou P-O, Donohue K (2011). Environment-dependent inbreeding depression: its ecological and evolutionary significance. New Phytol 189: 395-407.

Core Team R (2015). R: A Language and Environment for Statistical Computing. Available at http://www.r-project.org/.

Cotton PA (2006). Seasonal resource tracking by Amazonian hummingbirds. Ibis 149 : 135-142.

Crnokrak P, Roff DA (1999). Inbreeding depression in the wild. Heredity 83: 260-270.

Crow JF, Kimura M (1970). An Introduction to Population Genetics Theory. Harper Row: New York.

Economos AC, Lints FA (1985). Growth rate and life span in Drosophila. IV. Role of cell size and cell number in the biphasic relationship between life span and growth rate. Mech Ageing Dev 32: 193-204.

Enders LS, Nunney L (2012). Seasonal stress drives predictable changes in inbreeding depression in field-tested captive populations of Drosophila melanogaster. Proc $R$ Soc $B$ Biol Sci 279: 3756-3764.

Falconer DS, Mackay TFC (1996). Introduction to Quantitative Genetics, 4th edn. Pearson: Harlow.

Fox CW, Reed DH (2011). Inbreeding depression increases with environmental stress: an experimental study and meta-analysis. Evolution 65: 246-258.

Hedrick PW (1994). Purging inbreeding depression and the probability of extinction: full-sib mating. Heredity 73: 363-372.

Hoffmann AA, Parsons PA (1991). Evolutionary Genetics and Environmental Stress. Oxford University Press: Oxford.

Hoffmann AA, Willi Y (2008). Detecting genetic responses to environmental change. Nat Rev Genet 9: 421-432.

Johnson MD, Sherry TW (2001). Effects of food availability on the distribution of migratory warblers among habitats in Jamaica. J Anim Ecol 70: 546-560.

Koenker RW (2013). quantreg: Quantile Regression. R package version 5.05. http://cran. r-project.org/package=quantreg.

Koenker RW, D'Orey V (1987). Algorithm AS 229: computing regression quantiles. J R Stat Soc 36: 383-393.

Kristensen TN, Hoffmann AA, Pertoldi C, Stronen AV (2015). What can livestock breeders learn from conservation genetics and vice versa? Front Genet 6: Article 38.

Kristensen TN, Pedersen KS, Vermeulen CJ, Loeschcke V (2010). Research on inbreeding in the "omic" era. Trends Ecol Evol 25: 44-52.

Kristensen TN, Sørensen P, Pedersen KS, Kruhøffer M, Loeschcke V (2006). Inbreeding by environmental interactions affect gene expression in Drosophila melanogaster. Genetics 173: 1329-1336

Leroy G, Mary-Huard T, Verrier E, Danvy S, Charvolin E, Danchin-Burge C (2013). Methods to estimate effective population size using pedigree data: examples in dog, sheep, cattle and horse. Genet Sel Evol 45: 1

Markow TA, Raphael B, Dobberfuhl D, Breitmeyer CM, Elser JJ, Pfeiler E (1999). Elemental stoichiometry of Drosophila and their hosts. Funct Ecol 13: 78-84. 
Morton NE, Crow JF, Muller HJ (1956). An estimate of the mutational damage in man from data on consanguineous marriages. Proc Natl Acad Sci USA 42 855-863.

Norman JK, Sakai AK, Weller SG, Dawson TE (1995). Inbreeding depression in morphological and physiological traits of Schiedea lydgatei (Caryophyllaceae) in two environments. Evolution 49: 297-306.

Nunney L (1993). The influence of mating system and overlapping generations on effective population size. Evolution 47: 1329-1341.

Nunney L (1996). The response to selection for fast larval development in Drosophila melanogaster and its effect on adult weight: and example of a fitness trade-off. Evolution 50: $1193-1204$

Palstra FP, Ruzzante DE (2008). Genetic estimates of contemporary effective population size: what can they tell us about the importance of genetic stochasticity for wild population persistence? Mol Ecol 17: 3428-3447.

Reed DH, Fox CW, Enders LS, Kristensen TN (2012). Inbreeding-stress interactions: evolutionary and conservation consequences. Ann NY Acad Sci 1256: 33-48.

Schielzeth H, Forstmeier W (2009). Conclusions beyond support: overconfident estimates in mixed models. Behav Ecol 20: 416-420.

Schou MF (2013). Fast egg collection method greatly improves randomness of egg sampling in Drosophila melanogaster. Fly 7: 44-46.
Schou MF, Kristensen TN, Kellermann V, Schlötterer C, Loeschcke V (2014) A Drosophila laboratory evolution experiment points to low evolutionary potential under increased temperatures likely to be experienced in the future. J Evol Biol 27. 1859-1868.

Suzuki S (1998). Leaf phenology, seasonal changes in leaf quality and herbivory pattern of Sanguisorba tenuifolia at different altitudes. Oecologia 117: 169-176.

Sørensen JG, Loeschcke V (2004). Effects of relative emergence time on heat stress resistance traits, longevity and hsp70 expression level in Drosophila melanogaster J Therm Biol 29: 195-203.

Waller DM, Dole J, Bersch AJ (2008). Effects of stress and phenotypic variation on inbreeding depression in Brassica rapa. Evolution 62: 917-931.

Wang L, Clark AG (1995). Physiological genetics of the response to a high-sucrose diet by Drosophila melanogaster. Biochem Genet 33: 149-165.

Wang J, Hill WG, Charlesworth D, Charlesworth B (1999). Dynamics of inbreeding depression due to deleterious mutations in small populations: mutation parameters and inbreeding rate. Genet Res 74: 165-178.

White TCR (1993). The Inadequate Environment: Nitrogen and the Abundance of Animals. Springer Verlag: Berlin.

Zuur AF, leno EN, Walker NJ, Saveliev AA, Smith GM (2009). Mixed Effects Models and Extensions in Ecology with R. Springer: New York.

Supplementary Information accompanies this paper on Heredity website (http://www.nature.com/hdy) 